\title{
Acumulación y tramas agroalimentarias en América Latina
}

\author{
Roberto Bisang y Graciela E. Gutman
}

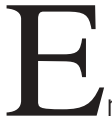

n el marco de la evolución de los mercados mundiales y de los nuevos modelos de apertura, varias producciones agroalimentarias en países del Mercosur mostraron en las últimas décadas un fuerte dinamismo, convirtiéndose en ejes de acumulación y crecimiento económico. La expansión productiva y los mayores niveles de competitividad estuvieron sustentados por la organización de estas producciones en tramas o complejos productivos; por el uso —con mínima adaptación local- de paquetes tecnológicos de origen externo, en el marco de la globalización de los nuevos paradigmas; por el surgimiento o consolidación de grupos acotados de grandes empresas en las etapas principa-

\section{Roberto Bisang}

Investigador-docente,

Instituto de Industrias,

Universidad Nacional de General Sarmiento

• rbisang@ungs.edu.ar

Graciela E. Gutman

Investigadora Principal,

Consejo Nacional de Investigaciones

Científicas y Técnicas (CONICET)

Profesora de la

Universidad de Buenos Aires

$\leadsto$ gutmang@fibertel.com.ar les de las tramas, y por una clara inserción en los mercados externos. Este artículo sostiene que la transnacionalización de segmentos y mercados de estos complejos afecta las posibilidades de desarrollo local o regional, entendido éste como la generación de tramas productivas localmente densas y diversificadas, con una distribución equitativa de rentas, ingresos y ganancias. 


\section{I}

\section{Introducción}

A partir de la década de 1990, en el marco de los procesos de apertura comercial y globalización de los mercados, los países del Mercosur ampliado ${ }^{1}$ han ido modificando sus patrones de especialización, orientándolos más hacia producciones basadas en la explotación de recursos naturales. La expansión productiva registrada en estos años estuvo sustentada en la difusión y adaptación de innovaciones tecnológicas, el surgimiento o consolidación de grupos acotados de grandes empresas en las etapas principales de las tramas productivas que articulan esas producciones, y una clara inserción en los mercados externos. Estos factores imprimieron un marcado dinamismo a las tramas y contribuyeron a modelar sus principales rasgos técnicos y productivos. Así, las tramas productivas aparecen como formas de organización impulsoras del crecimiento económico logrado en estas décadas, por su presunta capacidad de generar competitividad genuina. $^{2}$

Una revisión de los casos de más éxito en la región - particularmente las producciones agroalimentarias en Argentina, Brasil, Uruguay y, en menor medida, Paraguay y Bolivia - indica que la base de ese éxito ha sido la conformación de tramas productivas, es decir, de conjuntos articulados y coordinados de empresas con relaciones de largo plazo referidas a objetivos de interés común. Estas tramas permiten aunar recursos naturales abundantes y de calidad, competitividades individuales de cierta importancia y adecuados mecanismos de interrelación y articulación de actores y/o etapas hasta llegar a la demanda final.

La secuencia causal tras esta dinámica —que examinaremos en el presente trabajo- se sintetiza así: i) los países del Mercosur han redefinido parcialmen-

\footnotetext{
${ }^{1}$ Integrado por los países miembros del Mercosur (Argentina, Brasil, Paraguay y Uruguay), más Chile y Bolivia.

2 En este artículo entendemos por "competitividad genuina", desde la perspectiva de la economía en su conjunto, las ventajas de carácter estable en el tiempo que están asociadas a la incorporación de nuevos recursos naturales o humanos o al uso de tecnologías de producto o proceso y que se transforman en la base de ventajas competitivas para acceder a los mercados internacionales. Esta definición no toma en cuenta los efectos distributivos o redistributivos internos, y excluye las exportaciones sustentadas por subsidios (soportados por los consumidores y/o los contribuyentes), así como las basadas en la expoliación de recursos naturales y humanos.
}

te su patrón de especialización para orientarlo hacia un conjunto de actividades fuertemente competitivas, basadas en recursos naturales; ii) la expansión productiva estuvo sustentada por la adopción (con mínima adaptación local) de paquetes tecnológicos originados en el exterior, en el marco de los procesos asociados a la globalización de nuevos paradigmas productivos; iii) estos elementos se potenciaron a partir de una creciente tendencia a operar sobre la base de tramas productivas y de la generación de competitividades sistémicas.

Sin embargo, las potencialidades de las tramas productivas para sustentar procesos continuos de expansión dependen de sus características y configuración. En particular, sostenemos que, más allá de las posibilidades de valorizar los recursos naturales, el logro de ganancias conjuntas mayores (aunque asimétricamente distribuidas) depende de las estrategias de un pequeño conjunto de actores (por lo general grandes empresas transnacionales o locales) que coordinan la trama desde sus puntos nodales.

$\mathrm{Al}$ respecto, un número significativo de trabajos recientes sobre el tema aportan evidencias ${ }^{3}$ como las siguientes: i) las principales tramas agroalimentarias del Mercosur ampliado constituyen formas de organización empresarial eficientes para sustentar, de manera sistémica, el logro de mayores niveles de competitividad; ii) dentro de las tramas se reproducen y consolidan fuertes asimetrías entre las empresas participantes (asimetrías sustentadas en el control de activos estratégicos, económicos, financieros o tecnológicos), dando lugar a sistemas jerárquicos en los que algunas firmas ejercen el control y la coordinación del conjunto de empresas; y iii) salvo unos pocos casos, en estas actividades se observa un claro predominio de formas de capital concentradas y centralizadas, en particular filiales de capitales extranjeros (o empresas nacionales absorbidas por estos) o grupos económicos locales que controlan el conjunto productivo y parte significativa del diseño estratégico.

En función de esas evidencias, nuestra hipótesis central sostiene que, si bien las tramas agroalimentarias

\footnotetext{
3 CEPAL (1995), CNPq (1998), PROCISUR/BID (2000), CEPAL (2002) y Paulino y otros (2004).
} 
del Mercosur ampliado presentan un alto potencial de expansión productiva e inserción competitiva en los mercados mundiales, la transnacionalización de tramas y mercados importantes de estos complejos productivos afecta las posibilidades de desarrollo local o regional (entendido como la generación de tramas productivas localmente densas y diversificadas, con una distribución equitativa de rentas, ingresos y ganancias).
Por otra parte, si la organización de las actividades gira en torno a las tramas y estas, si bien generan excedentes, no garantizan automáticamente avances significativos en el conjunto de la economía, el paso siguiente es indagar - como lo hacemos en la parte final de este artículo- acerca de una necesaria reformulación de las políticas públicas, que brinde un marco regulatorio acorde con los objetivos de desarrollo económico y social.

\section{II}

\section{Tramas agroalimentarias y dinamismo competitivo de los países del Mercosur ampliado}

\section{Competitividad, empresas y tramas}

En años recientes, nuevos enfoques económicos han centrado su atención en que, en varias producciones agroalimentarias, han surgido tramas productivas en reemplazo del funcionamiento aislado de las empresas. El surgimiento o consolidación de tales tramas, en parte como respuesta a estrategias de externalización de procesos (impulsadas por la competencia interempresarial y el cambio tecnológico, con la consiguiente especialización en las actividades principales de la empresa), lleva a formas de coordinación vertical y horizontal basadas en contratos que favorecen la productividad conjunta y la competitividad internacional. ${ }^{4}$

El abordaje analítico de una trama productiva comprende diferentes pasos: i) la identificación de las relaciones técnicas y de las relaciones económicas al interior de la trama; ii) la individualización de las empresas nodales de la trama, esto es, aquellas con capacidad y poder económico, financiero o tecnológico para fijar criterios y coordinar el funcionamiento conjunto del complejo productivo; iii) el estudio de las formas de competencia predominantes en cada etapa y de los diferentes tipos de empresas participantes (tamaño, origen del capital, estrategias, organización empresarial); iv) el proceso de fijación de los objetivos comunes de la trama (acordados, inducidos o impuestos); v) las reglas de gobernanza del conjunto (las de mar-

\footnotetext{
${ }^{4}$ Dirven (1999), Granovetter (1985), Zylbersztajn y Farina (1997), Humphrey y Schmitz (2001), Albu (1999) y Ramos (1998).
}

co regulatorio público, las internas estipuladas por contratos formales o informales, incluidas aquellas sobre la distribución de beneficios y riesgos, etc.), y vi) las acciones y reacciones resultantes de interactuar con el entorno.

En este artículo nos centraremos principalmente en el análisis del perfil y comportamiento de los actores principales y en algunas reglas de gobernanza, aspectos clave para entender el proceso de conformación de jerarquías dentro de las tramas agroalimentarias y que explican las diferentes estrategias tecnoproductivas de los actores en las tramas bajo análisis.

Dado el objetivo central de las empresas - esto es, el logro de ganancias y renta o la búsqueda de competitividad en los mercados- la conformación de tramas productivas posibilita, en contextos de fuertes articulaciones verticales u horizontales, la cooperación en ámbitos específicos y la coordinación de los procesos, actividades y estrategias de las empresas e instituciones involucradas en la trama.

Las características de las empresas y otros agentes participantes (como los entes públicos o los consumidores), así como sus estrategias y las normas de regulación externas, contribuyen a explicar la existencia de jerarquías al interior de la trama. El poder económico, el acceso al financiamiento, las asimetrías tecnológicas y el control de los activos o de los conocimientos críticos - con sus distintas ponderaciones temporales - dan lugar a estas jerarquías. De esta manera se "establecen/ inducen/ejercen" asimetrías de poder al interior de la trama. Tales asimetrías permiten que las empresas más poderosas se apropien en mayor proporción de las mejoras sistémicas de productividad y ganancias, generando 
y reproduciendo capacidades diferenciadas de acumulación entre las empresas integrantes (proceso que suele agudizarse en períodos de crisis).

En cada uno de los segmentos productivos que constituyen la trama participan empresas que operan con: i) diversas capacidades tecnológicas, que se condicionan y complementan recíprocamente, si bien algunas de ellas son los principales factores determinantes de la calidad del producto, ${ }^{5}$ ii) capacidades financieras y económicas heterogéneas; iii) articulaciones diferenciadas con proveedores y con la demanda final e intermedia; iv) disparidades en los niveles y calidades de la información, y v) diferentes escalas económicas. Estas diferencias conducen a su vez a respuestas disímiles frente a idénticas modificaciones en el entorno.

Por otra parte, las distintas etapas de una trama pueden llevarse a cabo en diversos lugares geográficos (regiones o países), lo que afecta la localización del empleo, los ingresos u otras variables.

En la evolución de las tramas se puede identificar, en distintos momentos y etapas, aquellas empresas que operan como coordinadoras o centros de comando, constituyéndose así en nodos de la trama. El control de estos nodos determina las jerarquías internas, genera poder y sienta las bases para el reparto asimétrico de las rentas. Por esta razón son objetivo de los marcos regulatorios (Bisang, Gutman y otros, 2000).

$\mathrm{Al}$ estar regida la relación o articulación entre las empresas de la trama por contratos de carácter formal o (en la mayoría de los casos) informal, las empresas nodales establecen incentivos privados ${ }^{6}$ para alinear al complejo tras determinados objetivos, su logro y la posterior evaluación del desempeño conjunto. La aceptación de estos parámetros - ya sea consensuada, inducida, impuesta o por adhesión en un marco de escasas alternativas - cohesiona a la trama y le otorga

\footnotetext{
${ }^{5}$ Se comercializan en mercados concentrados, con fuertes asimetrías de información, y corresponden a productos y/o procesos muy específicos que inducen al uso de determinadas tecnologías complementarias a lo largo de la trama. Por ejemplo, las semillas transgénicas de soja resistentes al glifosato son altamente específicas en su diseño, predeterminan gran parte del paquete agronómico y sus tecnologías apropiadas (uso de siembra directa y glifosato) y del proceso de producción (tareas de presiembra, períodos de siembra y cosecha, sistemas de control de malezas e insectos, etc.). Se comercializan en mercados con oferta muy concentrada y fuertes asimetrías, tanto tecnológicas como de información e incluso económicas.

${ }^{6}$ El sistema de incentivos se basa en un precio de referencia corregido por esquemas de premios y castigos en función de atributos de calidad, tiempo de entrega, productividad industrial del insumo primario, y otras condiciones.
}

estabilidad temporal. Un requisito esencial de los contratos es la clara especificación de los procesos y del producto o servicio que se intercambiará. Las decisiones sobre quién, cómo, dónde y con qué criterios se fijan las normas técnicas, se convierten habitualmente en aspectos clave de la ulterior dinámica de las tramas.

El funcionamiento en tramas puede aumentar la eficiencia conjunta en algunas producciones y lograr, según los casos, reducir los costos de transacción (especialmente si se trata de bienes diferenciados; reducir al mínimo las fallas de información y coordinación de los mercados, tanto en consumo como en inversión; aumentar la escala en proyectos de alto riesgo; facilitar los procesos de generación y difusión de innovaciones; establecer mecanismos de distribución de riesgos, y formular estrategias más consistentes (minimizando los errores) para la evolución futura del conjunto de empresas.

Como resultado de estas dinámicas, dentro de las tramas productivas suelen generarse fuertes asimetrías (tecnológicas, económicas o financieras) que se traducen en repartos asimétricos de las rentas generadas por la trama en su conjunto. Los diferentes ritmos de acumulación de los diversos actores de la trama guardan una estrecha relación con sus características estructurales.

Como formas particulares de organización interempresarial, las tramas tienen particular importancia en las actividades agroalimentarias. Esto se debe, entre otras razones, a que permiten distribuir los riesgos asociados a los factores y procesos naturales y biológicos propios de estas producciones: entre otros, los imponderables climáticos; la autonomía de los ciclos biológicos (de cosechas, crianza pecuaria, fermentación y otros) que marcan el ritmo de los procesos de transformación; cierto grado de determinismo que impone la calidad de la materia prima en los posteriores procesos industriales, y los rasgos propios e idiosincrásicos de corte cultural o regulatorio que enmarcan la producción y el consumo de alimentos desde la logística que articula la comercialización al consumidor final (Gutman, 1999a). Estas características han quedado reflejadas tempranamente en la conformación de fuertes articulaciones interempresariales como sostén organizacional de estas producciones.

\section{Tramas agroalimentarias en el Mercosur am- pliado: evolución reciente y desafíos}

En este contexto, las principales tramas agroalimentarias de los países del Mercosur ampliado han adquirido 
cada vez mayor importancia desde comienzos del decenio de 1990, merced a un rápido crecimiento interno y una fuerte inserción internacional. Con diversos matices e intensidades, los aceites, productos lácteos, carnes, vinos, frutas y otros productos han exhibido cambios considerables respecto del pasado reciente en distintos países.

Varios aspectos son comunes: el crecimiento productivo y tecnológico; la presencia de agentes nuevos y renovados en los diversos estadios productivos, de comercialización y de provisión de insumos; una clara orientación exportadora $\mathrm{y}$, fundamentalmente, una creciente tendencia a la conformación de tramas (PROCISUR/BID, 2000).

El dinamismo reciente de algunas tramas agroalimentarias en el Mercosur corrobora las afirmaciones anteriores (cuadro 1).

Los casos de la trama láctea y la trama de oleaginosas son representativos de estas nuevas dinámicas. Inmersa en un proceso de fuerte reestructuración empresarial, de innovaciones en procesos y productos, y de una reorientación exportadora hacia el Mercosur, la trama láctea en Argentina creció entre 1992 y 1998, en su etapa primaria, a una tasa anual acumulativa y sostenida cercana al 7\%, luego de ha- ber exhibido un comportamiento cíclico y una tasa anual media inferior al $1 \%$ en los seis años anteriores; la producción industrial en 1992-1998 creció a una tasa anual de 12\% (Gutman, Guiguet y Lavarello, 2004; Gutman, 1999b). La expansión y reestructuración de este subsistema en Brasil, impulsado por la demanda interna, fue igualmente importante (Bortoleto y Wilkinson, 2000).

La producción, molienda y exportación de soja y sus subproductos conformó una de las tramas agroalimentarias más dinámicas de la región. A nivel primario la producción creció aceleradamente en Argentina, Brasil, Paraguay, Bolivia y, más recientemente, en Uruguay. En el caso de Argentina, esta expansión se sustentó en la adopción generalizada de un nuevo paquete tecnológico (semilla transgénica, siembra directa y fertilizantes) sobre la base de un modelo de organización productiva caracterizado por la separación entre los propietarios de las tierras y las empresas encargadas de llevar a cabo el proceso productivo, y por una creciente influencia de los proveedores de insumos (Bisang, 2003a y 2004). Los restantes países de la región siguen modelos similares, aunque en Brasil con restricciones al uso de semillas modificadas genéticamente.

CUADRO 1

Mercosur, Chile y Paraguay: Evolución productiva

de algunas tramas agroalimentarias

\begin{tabular}{|c|c|c|c|c|c|c|c|c|c|c|}
\hline \multirow[t]{2}{*}{ Trama } & \multicolumn{2}{|c|}{ Argentina } & \multicolumn{2}{|c|}{ Brasil } & \multicolumn{2}{|c|}{ Uruguay } & \multicolumn{2}{|c|}{ Chile } & \multicolumn{2}{|c|}{ Paraguay } \\
\hline & 1990 & 2002 & 1990 & 2002 & 1990 & 2002 & 1990 & 2002 & 1990 & 2002 \\
\hline $\begin{array}{l}\text { Leche } \\
\text { (producción en miles de litros) }\end{array}$ & 6281,0 & 8500,0 & 15075,0 & 22452,0 & 963,9 & 1431,2 & 1390,0 & 2180,0 & 225,0 & 375,0 \\
\hline $\begin{array}{l}\text { Leche en polvo } \\
\text { (exportación en miles de toneladas) }\end{array}$ & 14,0 & 136,0 & 0,01 & 1,30 & 2,21 & 29,05 & 1,60 & 9,98 & - & 0,14 \\
\hline $\begin{array}{l}\text { Carne } \\
\text { (producción en miles de toneladas) }\end{array}$ & 2650,0 & 2700,0 & 5008,0 & 7314,0 & 334,0 & 411,0 & 242,0 & 199,0 & 189,0 & 205,0 \\
\hline $\begin{array}{l}\text { Carne } \\
\text { (exportación en miles de toneladas) }\end{array}$ & 451,0 & 348,0 & 249,0 & 881,0 & 192,0 & 259,0 & 0,0 & 1,0 & 130,0 & 20,0 \\
\hline $\begin{array}{l}\text { Carne } \\
\text { (exportación en miles de dólares) }\end{array}$ & 158,0 & 160,0 & 49,0 & 430,0 & 132,0 & 148,0 & 0,0 & 1,0 & 97,0 & 13,0 \\
\hline $\begin{array}{l}\text { Vinos } \\
\text { (producción en miles de toneladas) }\end{array}$ & 1400,0 & 1200,0 & 310,0 & 320,0 & 94,0 & 71,0 & 390,0 & 570,0 & 7,0 & 6,0 \\
\hline $\begin{array}{l}\text { Soja en grano } \\
\text { (producción en millones de toneladas) }\end{array}$ & 11,0 & 35,0 & 19,8 & 42,1 & 0,0 & 0,1 & - & - & 1,8 & 3,3 \\
\hline $\begin{array}{l}\text { Complejo soja } \\
\text { (exportación en millones de dólares) }\end{array}$ & 2025,0 & 5026,0 & 2554,0 & 6009,0 & 6,0 & 10,0 & 0,0 & 3,0 & 246,0 & 417,0 \\
\hline
\end{tabular}

Fuente: Elaboración propia con datos de la Organización de las Naciones Unidas para la Agricultura y la Alimentación (FAO) y el Departamento de Agricultura de los Estados Unidos. 
La etapa industrial de la molienda de los granos oleaginosos en Argentina y Brasil (los dos principales países exportadores del complejo, tanto a nivel regional como mundial) acompañó esta expansión de la producción primaria con fuertes inversiones (en buena medida de empresas transnacionales) e incorporación de tecnología. Dichas inversiones colocaron a la industria de la molienda en estos dos países a niveles tecnológicos y de escala similares a los mejores estándares internacionales (Gutman y Lavarello, 2003; Gutman, 2000).

La producción de soja — fuertemente orientada a los mercados mundiales, con innovaciones en los procesos y técnicas productivas en el sector primario, y una mayor presencia de grandes empresas transnacionales en la molienda y comercialización de granos oleaginosos- se duplicó en Brasil entre 1990 y el 2002, mientras que en Argentina se triplicó en igual lapso, con una expansión correspondiente de la capacidad de molienda (Bisang, 2003b). En ambos países esto se reflejó en el aumento de las exportaciones.

Otras importantes tramas agroalimentarias del Mercosur registraron evoluciones similares a las de lácteos y soja; por ejemplo, la trama cerealera mostró una notable expansión y reestructuración de las cadenas agroalimentarias del trigo y el maíz (PROCISUR/BID, 2000; Lavarello, 2003).

En la producción de carne, más allá de los problemas sanitarios de fines del decenio de 1990, los países de la región han dado saltos cuantitativos y cualitativos de cierta relevancia. Si bien en varias de las tramas regionales persisten desajustes en esta actividad, no es menos cierto que existen subcircuitos articulados a través de contratos para segmentos de carnes de alta calidad que han mostrado un claro dinamismo. A nivel agregado, los casos de Uruguay (coloca más del 70\% de su producción en los mercados externos) y Brasil (que duplicó sus colocaciones externas en menos de un lustro) se cuentan entre los más dinámicos. Un caso particular lo constituye Chile, que pese a su escasa tradición productora en este rubro, se ha especializado en nichos de mercado de alto valor y está claramente orientado a la exportación. ${ }^{7}$

Otros ejemplos de las nuevas dinámicas regionales son las tramas avícola y de vinos. Mejor articulada y con una estabilidad de más largo plazo, la trama avícola muestra claros avances en la región, lo que ha

\footnotetext{
7 Bisang (2003c), Buxedas (2003), Paulino y otros (2004), Zylbersztajn y Pinheiro Machado (2000).
}

llevado en Argentina y Brasil a mejorar el abastecimiento del mercado local y a crecientes exportaciones.

El caso de los vinos se destaca por su evolución hacia productos de mayor valor agregado, en un proceso de reconversión que fue impulsado por importantes inversiones de capital regional y extrarregional. A los avances iniciales de los vinos chilenos se han ido sumando los de producciones argentinas y, para variedades específicas, uruguayas (Azpiazu y Basualdo, 2000; INTA, 2003).

El conjunto de las actividades descritas, más allá de la esperable heterogeneidad al interior de las tramas y entre ellas en los diversos países, presenta varios rasgos comunes en sus formas de organización y comportamiento tecnoproductivo. Si bien la articulación de las producciones en tramas ha permitido mejorar los procesos de generación de ventajas competitivas dinámicas, especialmente en el plano innovativo y comercial, el dinamismo productivo muestra algunos rasgos particulares.

En primer lugar, cabe señalar que en la mayoría de los casos las nuevas formas de organizar la producción elevan las escalas técnicas y económicas (tamaño mínimo de la explotación o de las plantas industriales, equipamiento, capacitación de la mano de obra). Tanto a nivel primario como industrial y comercial, estos avances requieren crecientes dotaciones de capital fijo y circulante. ${ }^{8}$ Por lo tanto, las exigencias asociadas a las nuevas tecnologías (en términos de capital fijo y/o circulante, y de habilidades empresariales o laborales mínimas) se traducen en posibilidades de acceso asimétricas de las empresas a las nuevas

\footnotetext{
${ }^{8}$ La adopción de un paquete tecnológico basado en siembra directa+biocidas+semillas transgénicas implica un capital adicional mínimo de unos 100.000 dólares, lo que hace inviable la integración vertical para productores agropecuarios con menos de 100 hectáreas, sobre todo si se consideran los endebles mercados de capital de los países analizados. Una tendencia similar se observa en la actividad láctea, donde la mecanización y las mejoras genéticas (y las tecnologías de proceso asociadas) elevan el umbral mínimo de las explotaciones, por lo cual se requiere no sólo mayor capital fijo sino también circulante. Y lo mismo se da en sectores industriales clave (como la molienda de oleaginosas o las plantas frigoríficas para exportación) o en la producción de insumos agropecuarios (desarrollo comercial de la genética vegetal o animal; producción de agroquímicos). A título de ejemplo, en Argentina el tamaño medio de una explotación lechera (tambo) pasó de 65,9 vacas a 145,1 vacas entre 1988 y 2000 . En el caso de la industria oleaginosa, el tamaño medio de una planta de molienda pasó de 1.100 toneladas diarias procesadas en 1990 a 2.300 toneladas diarias en 2003; en este último año, la mayor planta instalada en Argentina tenía una capacidad de procesamiento diario de 12.000 toneladas.
} 
técnicas, lo que acentúa el proceso de concentración productiva.

Las especificidades de algunas demandas finales, las variaciones en los precios internacionales y la escasa competitividad de los mercados financieros, son otros factores que refuerzan el proceso de diferenciación. Como resultado, existe una clara tendencia a la concentración y dualización de las estructuras productivas, al generarse dos circuitos diferenciados:

i) El circuito conformado por actividades de cierta escala, con mejoras técnicas continuas, controles de calidad y adecuación a los estándares internacionales, impulsado por la comercialización minorista concentrada ${ }^{9}$ cuando la producción se dirige al mercado interno, y por la dinámica de los mercados externos cuando la producción tiene un alto coeficiente de exportación, y dotado de grandes potencialidades para el aumento de la exportación, la producción y los rendimientos. Son estas tramas, por lo demás, las que tienen un impacto positivo en las cuentas externas (en el caso de Argentina, la oleaginosa, la cerealera, la vitivinícola y, en menor proporción, la láctea).

ii) El circuito centrado en productores agropecuarios y empresas industriales o comerciales (a las que en adelante llamaremos simplemente empresas), pequeños o fuertemente endeudados, con dificultades para efectuar una reconversión o acceder a las nuevas técnicas; por lo general, su actividad se circunscribe a mercados regionales o locales, con débiles estándares de calidad y bajas posibilidades de ingreso a círculos virtuosos de producción. Mayoritario en número de explotaciones agropecuarias y de empresas industriales, y de indudable impacto en el empleo, este circuito productivo se encuentra, en el mejor de los casos, en los umbrales mínimos para mantener la actividad, con bajas o nulas posibilidades de acumulación y desarrollo. ${ }^{10}$

En segundo lugar, la presencia creciente de nuevos actores (o el crecimiento de otros de larga data) en algunas de las actividades principales o nodos de las tramas conduce a una redistribución interna del

\footnotetext{
${ }^{9}$ Es decir, los grandes distribuidores minoristas, como supermercados, hipermercados y similares.

${ }^{10}$ Como consecuencia, en los países analizados existen circuitos marginales de producción láctea a nivel primario, que se articulan con pequeñas fábricas, casi artesanales, y concluyen abasteciendo a segmentos de mercados locales o regionales, con estándares técnicos más bajos y reducidas posibilidades de acumulación. Algo similar sucede en los circuitos de la carne y de la elaboración de farináceas.
}

poder en cuanto a quién decide qué producir, cómo y para qué destino. En ese sentido, tanto los proveedores de insumos para el agro como los grandes distribuidores minoristas tienden a establecer nuevos espacios de poder que disputan los niveles de acumulación del conjunto (Gutman, 2002). En ambos casos, estos procesos de reconfiguración fueron acompañados por mayor concentración y transnacionalización de los mercados. ${ }^{11}$ Tal evolución estuvo unida a frecuentes tensiones entre productores agropecuarios y empresas que operan en distintas etapas dentro de la trama y, simultáneamente, a un claro dinamismo tecnoproductivo.

La oleada de inversiones externas hacia los países de la región en el decenio de 1990 reforzó estos procesos. Las inversiones extranjeras directas (IED) - atraídas por las nuevas condiciones regulatorias y el Mercosur ampliado, y en el marco de estrategias de expansión globalizada- se focalizaron en algunos segmentos industriales, especialmente en la provisión de insumos, en el procesamiento industrial y en la gran distribución minorista. Ceñidas a estrategias globales de crecimiento basadas en avances técnicos que requieren actividades de gran escala, ingresaron a un rápido proceso de concentración o de alianzas estratégicas, lo que condujo de hecho a estrategias de expansión de corte regional. ${ }^{12}$

De esta forma, las tramas más dinámicas exhibieron en forma simultánea fuertes transformaciones tecnoproductivas, el establecimiento o consolidación de nuevas formas de organización (tramas), un crecimiento impulsado por los mercados externos, y la reconfiguración de las jerarquías y de los nodos o mandos dentro de las tramas. Todo esto lleva a replantear las reglas de gobernanza, las jerarquías y las asimetrías de poder en las tramas con capacidad de acumulación (Wilkinson, 2002).

\footnotetext{
${ }^{11}$ La evolución del comercio minorista de alimentos en Argentina es reveladora de estos procesos: en el año 2000, el estrato de hipermercados y supermercados alcanzaba a más del 50\% de las ventas de alimentos; conformado por 77 empresas en cadena, las ventas de las siete mayores alcanzaban al $78 \%$ de las ventas del estrato. Cuatro quintos de este porcentaje correspondían a filiales de empresas transnacionales (Gutman, 2000).

12 En el marco de la afluencia de inversiones externas a estos países, en la década de 1990 ingresaron a ellos o ampliaron considerablemente sus actividades casi todos los oferentes mundiales de semillas (Monsanto, Bayer Agrocrop Science, Syngenta, Hoescht, Cargill, Nidera, Ishiara, Dow Chemical, ICI, Bunge, Novo Hydro, ABS Genetics; USA Genetics, Nestlé, Danone, Parmalat, Unilever, CPC USA, PEPSICO, y las cadenas de comercialización Ahold, Walt Mart y Carrefour/PROMODEs, entre otros).
} 


\section{Cambios en las jerarquías y en las estrategias de las empresas}

Los cambios en el contexto institucional y regulatorio de la década de 1990 en los países del Mercosur ampliado y en los mercados mundiales, impulsaron nuevas formas de gobernanza de las principales tramas agroalimentarias, sustentadas en una mayor presencia de capital transnacional, el surgimiento y consolidación de agentes y un nuevo conjunto de regulaciones públicas concordantes con la desregulación de los mercados y la apertura comercial. Las nuevas reglas de gobernanza de las tramas agroalimentarias tendieron a modelarse a partir de:

- cambios en la estructura de proveedores, dado que la apertura comercial favoreció las importaciones de bienes de capital e insumos; ${ }^{13}$

- la presencia de fuertes inversiones externas realizadas bajo una lógica de complementación internacional, lo que modificó la dinámica operativa de varias de estas tramas;

- modificaciones en el marco legal, en particular en la ley de inversiones extranjeras, que facilitaron esa reconfiguración y garantizaron un trato más benévolo a esas inversiones y menores restricciones a la repatriación de rentas de empresas extranjeras;

- políticas tecnológicas centradas esencialmente en una amplia incorporación de equipamiento importado, con escasas restricciones a los flujos de tecnología y de capital (SEPCyT, 2003);

- la eliminación (en Argentina y otros países) de los mecanismos de regulación de algunas actividades productivas (en Argentina, Junta Nacional de Granos, Junta Nacional de Carnes, Comisión de Concertación Láctea y otras) y su reemplazo por la presión competitiva de los mercados externos. En este marco, se modificaron las jerarquías internas de varias de las tramas más dinámicas en la región, lo que se manifestó de varias formas. Ante todo, en la presencia generalizada de los principales proveedores internacionales de insumos para las produccio-

\footnotetext{
${ }^{13}$ El grueso de las empresas argentinas de biocidas fue absorbido por un número acotado de empresas internacionales líderes (Monsanto, Syngenta, Dow), las que además de ampliar sus instalaciones productivas, establecieron redes comerciales de cobertura nacional (Bisang, 2003b). La industria local de maquinaria y bienes de capital fue desplazada del mercado por los grandes proveedores internacionales; en el caso del complejo oleaginoso ingresaron Alfa Laval, De Smet, Buhler, y Crown, entre otros (Gutman y Lavarello, 2003).
}

nes primarias de las cadenas agroalimentarias; en estos casos, la supremacía respecto de oferentes privados locales e incluso de los entes públicos de investigación y desarrollo (I+D) se basó en cierta preeminencia tecnológica (caso de los transgénicos), unida a un fuerte dominio financiero. $\mathrm{Al}$ mismo tiempo, inversiones externas que aportaban alta tecnología y estrechas relaciones internacionales a algunas fases clave de la etapa industrial, se sumaron al capital local de un número acotado de grupos económicos, los que rápidamente adoptaron estrategias de internacionalización. El reordenamiento de las jerarquías en estos casos obedeció al dominio tecnológico y económico, y al acceso a mercados internacionales de gran escala, en el marco de tramas que operan en mercados globalizados. A ello se sumó, como se dijo más atrás, la fuerte irrupción de los grandes distribuidores minoristas como nuevos actores de peso en la mayoría de las tramas.

Diversas son las razones que instalaron a estos últimos actores como nuevos nodos de las tramas: en particular, su poder financiero y su fácil acceso a las fuentes internacionales de financiamiento; su estratégica posición de mercado para captar e impulsar cambios en las pautas de consumo y hábitos de compra de la población; su control de áreas clave, como la logística asociada con la distribución; y, en especial, la posibilidad de arbitrar los desfases de precios y calidades entre mercados internos e internacionales en el marco de los procesos de apertura (Gutman, 2002).

Como se desprende de lo anterior, la innovación y el cambio tecnológico fueron los ejes principales de la reconfiguración de las jerarquías y, con ello, de las posibilidades de reposicionarse en las tramas, generando en ellas severas asimetrías entre productores agropecuarios y empresas. En tal sentido, las estrategias de las firmas líderes en los distintos mercados, asentadas en importantes innovaciones tecnológicas y organizativas, se centraron sobre todo en:

- la relocalización de actividades y la apertura de nuevas plantas;

- un mayor control sobre la materia prima, tanto en términos de cantidad como de calidad;

- el establecimiento de relaciones más estrechas (contratos o cuasi contratos) con proveedores y clientes;

- avances logísticos y comerciales;

- estrategias de diferenciación y diversificación productivas en las industrias productoras de bienes finales;

- externalización de funciones y actividades y refocalización en las actividades centrales (core activities); 
- articulación de los procesos productivos para el logro conjunto de mayor productividad (eficiencia), calidad y seguridad alimentaria (estableciendo sistemas de control de calidad y sistemas de identidad preservada y/o trazabilidad);

- absorción de firmas locales, y de sus canales de proveedores y de distribución, por parte de empresas extranjeras;

- formulación de estrategias a escala regional que incluyan la distribución regional de etapas y segmentos de la cadena agroalimentaria, el reparto de los mercados, y la coordinación regional y global entre grandes empresas transnacionales que actúan en las diferentes etapas;

- desarrollo de tramas empresariales y actividades conjuntas en el ámbito productivo, comercial y tecnológico (Bisang y Gutman, 2001).

Lo que hemos descrito pone el tema de las asimetrías tecnológicas en el centro de la relación "jerarquía en las tramas-poder-acumulación”. Desde tal perspectiva, examinaremos el perfil de la oferta tecnológica de las principales tramas agroalimentarias bajo análisis.

\section{La oferta de tecnología de las principales tra- mas agroalimentarias del Mercosur ampliado}

En la región, las principales tramas agroalimentarias exhiben una amplia heterogeneidad, tanto entre los agentes que participan en la oferta tecnológica, como en la naturaleza de los acervos tecnológicos (tácitos y explícitos) que se difunden.

Así, formas artesanales de producción de baja escala (en establecimientos cercanos al nivel de subsistencia y con mínimas posibilidades de reproducción del capital), conviven con emprendimientos de gran escala que utilizan técnicas productivas de última generación (Bisang, Gutman y otros, 2000).

La identificación de quiénes y cómo lideran estos procesos dentro de cada una de las tramas productivas más dinámicas es esencial para analizar el aporte de estas formas organizativas al proceso local de acumulación.

Desde este punto de vista, y a nivel primario, ha habido en los últimos años cambios de significación en el perfil de la oferta, en medio de un rápido proceso de actualización innovativa. Las mejoras en las semillas (introducción de transgénicas y otras técnicas), la genética animal, el uso de nuevas técnicas de cultivo (como labranza cero y rotaciones complementarias entre cultivos) y el uso más intensivo de herbicidas y biocidas, van perfilando un nuevo paradigma tecnológico, que diversos autores llaman la agricultura dominada por el conocimiento (Cap, 1997).

Como se dijo antes, las modificaciones tecnoproductivas al interior de las tramas, además de generar dos circuitos productivos, uno de gran escala y orientación externa y otro pequeño/mediano de orden local o regional, otorgaron un mayor protagonismo a las grandes empresas en virtud de la escala de sus actividades.

El proceso de concentración circunscribió las jerarquías más altas de las tramas agroalimentarias a un número acotado de actores: empresas transnacionales; cooperativas, y grupos económicos o grandes empresas de capital nacional. Su presencia en cada trama varía según la actividad y el país. En Chile, la industria láctea tiene fuerte presencia internacional, mientras que en Uruguay y Paraguay se centra en dos cooperativas; en Argentina y Brasil existe cierto equilibrio entre grandes empresas de capital local y filiales de empresas transnacionales. La presencia de capital externo en la industria de la carne está limitada a nichos o procesos particulares en la mayoría de los países; por el contrario, en la industria de vinos hay una fuerte presencia internacional, al igual que en la molienda industrial de granos oleaginosos (más allá de unos pocos pero significativos grupos empresariales de capital local).

Sin embargo, esto no se traduce necesariamente en conductas tecnológicas similares de los principales actores. Tanto las cooperativas como las grandes empresas de capital nacional exhiben en general un muy bajo dinamismo tecnológico, como muestran datos recientes para Argentina y Uruguay (Gutman y Cesa, 2002). Este hecho suele traducirse en una pérdida de jerarquía frente al predominio técnico de empresas transnacionales con capacidades propias de efectuar avances técnicos y, por ende, llevar a su venta o a la realización de alianzas estratégicas.

$\mathrm{Al}$ respecto, cabe referirse al comportamiento de los institutos públicos de investigación, muy heterogéneos en cuanto a escala de actividades, antigüedad, perfiles de especialización y recursos humanos y económicos. La mayoría de ellos tendió a adaptarse a las nuevas circunstancias. Pero más allá de su aporte a las formación de capacidades locales, su desempeño se vio dificultado por i) fuertes desarticulaciones entre distintos institutos públicos de investigación que se dedican a aspectos parciales, pero sin contar con estrategias globales para crear activos críticos en todas las tramas; ii) la inercia de programas de trabajo centrados en 
problemas del viejo modo de producción, que a menudo se convierte en una barrera para acceder a procesos de innovación actualizados,${ }^{14}$ iii) severos problemas presupuestarios asociados con la crisis financiera de los Estados nacionales, especialmente a partir de la segunda mitad de la década de 1990, que llevó a recortes presupuestarios para los institutos de investigación y desarrollo (PROCISUR, 2002; Lindarte, 1994).

La debilidad de estos institutos y la fortaleza de las empresas transnacionales tornan muy importantes las actividades de las instituciones públicas de ciencia y tecnología, especialmente las de mayor envergadura que se orientan a la investigación básica. En este sentido, planteamos la necesidad de repensar - a nivel centrallas intervenciones públicas en estas actividades.

A partir de este perfil de comportamiento de los principales actores de las tramas, se han introducido cambios en la oferta tecnológica de las principales tramas, oferta liderada por empresas internacionales provenientes del campo industrial y favorecidas por los procesos de apertura de la economía. Esto se comple- mentó, en todas las tramas agroalimentarias analizadas, con la presencia de oferentes locales de peso decreciente que incluyen empresas privadas e institutos públicos de investigación agrícola (Bisang, Gutman y otros, 2000). Por su parte, el funcionamiento en tramas facilitó el proceso de difusión y adopción de nuevos paquetes tecnológicos.

Contribuyeron al proceso de difusión de las nuevas tecnologías algunas variables del mercado (entre otras, movimientos favorables de los precios, demandas particulares y selectivas provenientes de la comercialización) y el marco institucional de las tramas, donde los contratos de provisión entre la industria y los productores agropecuarios y entre los productores agropecuarios y los grandes distribuidores minoristas impulsaron estos cambios.

El cuadro 2 indica que, tanto en la provisión de insumos a las actividades primarias como en las actividades industriales, gran parte de las principales tecnologías se concentra en un acotado número de empresas transnacionales.

CUADRO 2

Argentina, Brasil y Uruguay: Principales proveedores de equipos e insumos tecnológicos en algunas tramas agroalimentarias

\begin{tabular}{llll}
\hline Insumos/proveedores & Uruguay & Argentina & Brasil \\
\hline $\begin{array}{l}\text { Semilla de soja transgénica } \\
\text { (variedad RR) }\end{array}$ & Nidera $^{\mathrm{a}}$ & Nidera $^{\mathrm{a}}$ & No aprobado para uso \\
& Don Mario & Don Mario & comercial $^{\mathrm{b}}$ \\
& Relmó $^{\mathrm{b}}$ & Relmó $^{\mathrm{b}}$ & Otros
\end{tabular}

Semilla de soja no transgénica

Maíces transgénicos

(variedad bt)

Maíces híbridos
Pioneer $^{\mathrm{a}}$

Dekalb $^{\mathrm{a}}$
Agroceres/Monsanto ${ }^{\mathrm{a}}$

Fund Estaduales/EMBRAPA ${ }^{\mathrm{c}}$

Sementes do Brasil ${ }^{\mathrm{a}}$

Otros

Monsanto Pioneer ${ }^{\mathrm{a}}$

Monsanto Dekalb

Don Mario ${ }^{b}$

Monsanto Pioneer ${ }^{\mathrm{a}} 70 \%$

\author{
Agroceres/Monsanto ${ }^{\mathrm{a}}$ \\ $32 \%$ \\ Carguil $^{\text {a }} 25 \%$ \\ Pioneer $^{\mathrm{a}} 13 \%$ \\ Unimilho/EMBRAPA $^{\mathrm{c}} 12 \%$ \\ Sta. Helena Sementes Ltda.
}

\footnotetext{
${ }^{14}$ A nivel primario, en los principales institutos públicos de investigación —el Instituto Nacional de Tecnología Agropecuaria (INTA) en Argentina, la Empresa Brasileña de Investigación Agropecuaria (EMBRAPA), el Instituto de Investigaciones Agropecuarias (INIA) en Chile y en Uruguay - los programas de actividades tienen una fuerte impronta del pasado. Así, existe una marcada tendencia a corregir
}

factores que limitan no tanto la calidad, sino la cantidad producida, lo que influye en la posterior etapa industrial. En otro orden, y más allá de las políticas recientes, las preocupaciones tienden a centrarse en problemas técnicos de producción dentro del predio, sin mayores relaciones con la trama en su conjunto. 
(continuación)

\begin{tabular}{|c|c|c|c|}
\hline Insumos/proveedores & Uruguay & Argentina & Brasil \\
\hline Semilla de trigo & $\begin{array}{l}\text { INIA }^{\mathrm{c}} \\
\text { Buck-ACA y otros }^{\mathrm{a}} \\
\text { Pioneer }^{\mathrm{a}}\end{array}$ & $\begin{array}{l}\text { Bioceres/INTA } \\
\text { Buck } \\
\text { Klein } \\
\text { INTA }^{\mathrm{c}}\end{array}$ & EMBRAPA $^{\mathrm{c}}$ \\
\hline Tractores & & $\begin{array}{l}\text { Agrinar }^{\mathrm{a}} \\
\text { John Deere } \\
\text { Zanello } \\
\text { Pauny }\end{array}$ & $\begin{array}{l}\text { Valmet/Valtra } \\
\text { CATERPILLAR }^{\mathrm{a}} \\
\text { Massey Fergusson }^{\mathrm{a}} \\
\text { FIAT }^{\mathrm{a}} \\
\text { Deutz-Fahr }^{\mathrm{a}} \\
\text { SLC John Deere }\end{array}$ \\
\hline Cosechadoras & & $\begin{array}{l}\text { Vasalli/Don Roque } \\
\text { John Deere } \\
\text { Class }^{\mathrm{a}}\end{array}$ & $\begin{array}{l}\text { Class }^{\mathrm{a}} \\
\text { John Deere }^{\mathrm{a}} \\
\text { AGCO do Brasil }^{\mathrm{a}} \\
\text { Agrale Deutz-Fahr }^{\mathrm{a}}\end{array}$ \\
\hline $\begin{array}{l}\text { Sembradoras de siembra } \\
\text { directa }\end{array}$ & $\begin{array}{l}\text { Bertini }^{\mathrm{a}} \\
\text { Agrometal }^{\mathrm{a}} \\
\text { Mainero }^{\mathrm{a}} \\
\text { Apache }^{\mathrm{a}} \\
\text { Otras }\end{array}$ & $\begin{array}{l}\text { Bertini } \\
\text { Agrometal } \\
\text { Mainero } \\
\text { Apache } \\
\text { Otras }\end{array}$ & $\begin{array}{l}\text { Yanmar do Brasil } \\
\text { Massey Ferguson Brasil } \\
\text { John Deere }^{\mathrm{a}}\end{array}$ \\
\hline Pulverizadoras & & $\begin{array}{l}\text { Pla } \\
\text { Metalfor } \\
\text { Otros }\end{array}$ & \\
\hline Glifosato & & $\begin{array}{l}\text { Monsanto }^{\mathrm{a}} \\
\text { PASA }^{\mathrm{a}} \\
\text { ATANOR }^{\mathrm{a}}\end{array}$ & \\
\hline Urea & & Profertil $^{\mathrm{a}}$ & $\begin{array}{l}\text { Petrobrás } \\
\text { Ultrafértil }^{\mathrm{a}}\end{array}$ \\
\hline Otros agroquímicos & Hydro Agri ${ }^{a}$ & $\begin{array}{l}\text { Dow }^{\mathrm{a}} \\
\text { Dupont }^{\mathrm{a}} \\
\text { Bayer }^{\mathrm{a}} \\
\text { Monsanto }^{\mathrm{a}} \\
\text { Atanor } \\
\text { YPF }^{\mathrm{a}} \\
\text { MOSAIC } \\
\text { Bunge }\end{array}$ & $\begin{array}{l}\text { Produquímica } \\
\text { Serrana fertilizantes } \\
\text { Norsk Hydro }^{\mathrm{a}} \\
\text { Cargill }^{\mathrm{a}} \\
\text { Copebrás } \\
\text { Fosfértil }\end{array}$ \\
\hline $\begin{array}{l}\text { Genética y reproducción } \\
\text { animal }\end{array}$ & $\begin{array}{l}\text { Cabañas privadas } \\
\text { ABS }^{\mathrm{a}}\end{array}$ & $\begin{array}{l}\text { Cabañas privadas } \\
\text { CIALE/La Elisa }_{\text {ABS }^{\mathrm{a}}} \\
\text { Alta Genetic }^{\mathrm{a}} \\
\text { Bovine Elite Inc }\end{array}$ & $\begin{array}{l}\text { ABS }^{\mathrm{a}} \\
\text { Alta Genetic }^{\mathrm{a}} \\
\text { Bovine Elite Inca }^{\mathrm{a}}\end{array}$ \\
\hline Sanidad animal & $\begin{array}{l}\text { Lab. Santa Elena } \\
\text { Bayer }^{\mathrm{a}} \\
\text { Novartis }^{\mathrm{a}} \\
\text { Hoechts }^{\mathrm{a}} \\
\text { Glaxo }^{\mathrm{a}} \\
\text { Pfizer }^{\mathrm{a}}\end{array}$ & $\begin{array}{l}\text { Biogénesis } \\
\text { Lab. San Jorge Bago } \\
\text { Rosenbush } \\
\text { Bayer } \\
\text { Novartis }^{\mathrm{a}} \\
\text { Hoechts }^{\mathrm{a}} \\
\text { Glaxo }^{\mathrm{a}} \\
\text { Pfizer }^{\mathrm{a}}\end{array}$ & $\begin{array}{l}\text { Bayer }^{\mathrm{a}} \\
\text { Ciba }^{\mathrm{a}} \\
\text { Novartis }^{\mathrm{a}} \\
\text { Hoechts }^{\mathrm{a}} \\
\text { Glaxo }^{\mathrm{a}} \\
\text { Pfizer }^{\mathrm{a}}\end{array}$ \\
\hline $\begin{array}{l}\text { Equipos de ordeña para el } \\
\text { tambo }\end{array}$ & & Alfa Laval ${ }^{\mathrm{a}}$ & \\
\hline $\begin{array}{l}\text { Equipos para molienda por } \\
\text { solvente de granos } \\
\text { oleaginosos }\end{array}$ & & $\begin{array}{l}\text { De Smet } \\
\text { Crown } \\
\text { Buhler }^{\mathrm{a}}\end{array}$ & $\begin{array}{l}\text { De Smet } \\
\text { Crown } \\
\text { Buhler }^{\mathrm{a}}\end{array}$ \\
\hline Envases especiales & $\begin{array}{l}\text { Tetrapack }^{\mathrm{a}} \\
\text { American Plast }^{\mathrm{a}}\end{array}$ & Tetrapack $^{\mathrm{a}}$ & Tetrapack $^{\mathrm{a}}$ \\
\hline
\end{tabular}

Fuente: Bisang, Gutman y otros (2000).

a Empresa transnacional. b Bajo licencia Monsanto. c Organismo público. 
Estas tendencias van conformando un nuevo modelo de innovación, caracterizado por:

- una creciente importancia de las tecnologías de la información y la biotecnología, que adquieren valor estratégico en la etapa primaria (sobre todo respecto a semillas y genética animal para lácteos y carnes), en el procesamiento industrial posterior y en la comercialización; en la etapa predomina el peso de los oferentes de insumos, el grueso de los cuales proviene del campo industrial;

- la redefinición de los nodos a partir de los cuales se generan impulsos innovativos; en tal sentido, se destaca el rol de los proveedores de insumos, sobre la actividad primaria y las fases industriales, y de los grandes distribuidores minoristas sobre la industria (en este caso a través de los requisitos técnicos incorporados en los mecanismos de aprovisionamiento);

- el aumento y la concentración de la oferta de las tecnologías principales en pocas empresas privadas, principalmente transnacionales líderes de alcance regional. Las actividades centrales de investigación y desarrollo de estas empresas se concentran casi con exclusividad en sus casas matrices; en el ámbito local estas actividades son menores, y, por lo general, de tipo adaptativo a las condiciones edafológicas y climáticas locales y al perfil del consumidor local. Las excepciones a esta situación son algunos avances llevados a cabo por institutos nacionales de investigación y unas pocas empresas privadas locales, en actividades muy acotadas (por ejemplo, en el desarrollo de semillas híbridas);

- el reordenamiento de las jerarquías internas en las tramas en virtud del dinamismo de algunos agentes y su predominio en el proceso de acumulación, con lo cual se establece en ellas un mecanismo de asimetrías tecnológicas. Se tiende a conformar paquetes tecnológicos que dominan en las distintas tramas agroalimentarias. Se trata de funciones de producción (de diversos productores agropecuarios) que son coordinadas a partir de un número reducido de tecnologías principales. Estas funciones de producción se componen además de una variada gama de tecnologías complementarias que - aun en su diversidad - son articuladas por las tecnologías principales, que les imprimen una determinada dirección. ${ }^{15}$ De esta forma, las tec-

${ }^{15}$ Entre las tecnologías principales, son paradigmáticos los casos de las semillas genéticamente modificadas, determinadas varieda- nologías principales tienden a modelar el paquete tecnológico del productor agropecuario e inciden sobre su posterior articulación con otras tecnologías.

Los paquetes tecnológicos que se conforman y su difusión presentan algunos rasgos comunes en el sector agropecuario:

- La generación y difusión de la tecnología se hacen cada vez más en paquetes tecnológicos elaborados por distintos agentes públicos y privados. El grado de codificación del paquete es cada vez mayor, y los espacios para la adaptación de la tecnología quedan acotados. ${ }^{16}$ Esta adaptación requiere elevados niveles de capacitación del productor rural; lleva a replantear tanto el perfil de los empresarios como el de las organizaciones que adaptan y difunden tecnología, e induce indirectamente a modificar la relación público/privada y a revisar la acción del sector público.

- El grado de codificación de las tecnologías (y de los paquetes tecnológicos) está muy asociado a la genética, tanto vegetal como animal. Se observa un creciente grado de especificidad en algunas tecnologías que componen los paquetes de la etapa primaria, sobre todo en cuanto a entornos productivos, escalas de producción y características de la materia prima producida. ${ }^{17}$

- Hay un aumento paulatino de la intensidad de la interacción entre los paquetes tecnológicos de la fase primaria y la fase industrial, motivada tanto por el sistema de precios como por las demandas del consumo final. Esto lleva a las empresas, por un lado, a implementar sistemas de trazabilidad, $\mathrm{y}$, por otro, a reformular las estrategias de relaciones en el marco de tramas más amplias. ${ }^{18}$

- En los procesos de generación, difusión e innovación tecnológicas se observa una progresiva

des vegetales y ciertos tipos de genética animal, los que van acompañados de escalas y tecnologías de procesos particulares en la producción primaria (por ejemplo, sistemas de siembra, uso de biocidas, fertilizantes y riego, modalidad de cosecha y tipo de alimentación del ganado), y a veces, en el procesamiento industrial.

16 Sin embargo, para introducir nuevas variedades vegetales o genéticas, es preciso adaptarlas a las condiciones locales, lo que abre un amplio espacio a la actividad de adaptación.

17 Tipos diferenciados de harinas, composición química de los aceites vegetales y la leche y cortes de carne vacuna, entre otras características.

18 Empresas que antes eran estrictamente industriales comenzaron no sólo a incursionar en biotecnología y genética sino a tener posiciones de liderazgo en ellas, y también a comercializar productos finales en el extremo de la cadena agroalimentaria. 
configuración de tramas más complejas, formadas por distintos agentes públicos y privados. Estos últimos exhiben un importante liderazgo tanto en diversas áreas de investigación y desarrollo -algunas de las cuales eran patrimonio tecnológico casi exclusivo del sector público en el modelo anterior - como en los procesos de difusión de tecnologías;

- Hay una trama de difusión de las innovaciones, con fuerte peso del capital privado, que se articula a partir de las redes de comercialización de los proveedores de insumos o de las relaciones establecidas entre los productores agropecuarios y los grandes distribuidores minoristas.
En el marco de este modelo de innovación, con las variantes registradas en los países del Mercosur, la presencia de formas de articulación interempresarial basadas en el control de determinadas tecnologías coloca a los oferentes de estas últimas en una importante posición estratégica. Quienes controlan dichas tecnologías pasan a ocupar roles de importancia en la jerarquía de las tramas, y a incidir sobre el sentido y características del proceso de acumulación. ${ }^{19}$ La estrategia del conjunto queda fuertemente condicionada/ inducida por los agentes que dominan la generación y difusión de las tecnologías principales. Una parte importante de ellas es desarrollada actualmente por capital privado transnacional. ${ }^{20}$

\section{III}

\section{Tramas, acumulación e instituciones}

A partir de esta lógica de funcionamiento del sistema (un conjunto acotado de empresas agroalimentarias con capacidad de acumulación basada en el control de nodos en las tramas, recursos primarios y tecnología), cabe preguntarse cuál es el rol estratégico de las instituciones públicas.

Se puede suponer que un objetivo importante de tales instituciones es contribuir a que las tramas más dinámicas se radiquen en el territorio local de manera que se potencie el efecto de derrame sobre el resto de las actividades y se logre una redistribución equitativa de rentas entre los agentes participantes. Siendo así, existen al menos dos planos analíticos: el primero se refiere a la estrategia más adecuada para alcanzar tales fines y el segundo a los instrumentos operativos.

A nivel estratégico, si la acumulación se sustenta en el hecho de que en la jerarquía de la trama ocupen altas posiciones empresas que tienen clara preeminencia en la oferta de las tecnologías principales, las acciones de la institucionalidad pública deben centrarse en esas actividades. Las políticas públicas deberán dirigirse prioritariamente a la generación, adaptación, apropiación y difusión (en este último caso mediante la formulación de normas de contexto) de las tecnologías principales de cada una de las tramas productivas. Desde esta perspectiva, las instituciones de ciencia, tecnología e innovación resultan actores clave en la aplicación de las estrategias.

En el marco de la estrategia descrita, es necesario ajustar los instrumentos tradicionales de política pública para el logro del objetivo general. Se trata no sólo de aquellos destinados a regular los niveles de beneficio de las empresas (normas impositivas, de competencia y otras), sino también de los que cobran relevancia al considerar a la trama como objeto de regulación para las políticas públicas:

i) el conjunto de normas (sanitarias, bromatológicas, de contenido, etc.) que definen la calidad de los productos generados en las diversas tramas;

ii) los requerimientos mínimos de calidad y seguridad que rodean los procesos de producción;

iii) las políticas impositivas (incluidas las arancelarias) que modifiquen los precios relativos, favoreciendo el desarrollo local de etapas de mayor valor;

iv) las restricciones a la libre circulación de capital, como contrapartida a la obtención local de rentas netas asociadas a posiciones jerárquicas en las tramas;

v) políticas de arbitraje y control de las relaciones entre las diversas etapas que conforman la trama. En otro plano y desde una perspectiva de mayor

\footnotetext{
${ }^{19}$ En este proceso existe una constante tensión entre diversos agentes por el control de las redes y la apropiación de los excedentes, que se manifiesta, por ejemplo, en las pugnas entre algunos estratos de la industria y la comercialización o entre la industria y la producción primaria.

${ }^{20}$ Los recientes procesos de fusiones y adquisiciones lideradas por unas pocas empresas internacionales (Monsanto, Syngenta, Bayer, Dow y otras) en el caso de las semillas transgénicas, es ilustrativo de este comportamiento.
} 
alcance, otro conjunto de políticas (crediticias, impositivas, etc.) debería apuntar al desarrollo tecnológico y productivo, en manos de empresas y/o instituciones lo-

\section{IV}

\section{Conclusiones}

En las últimas décadas, algunas producciones basadas en recursos naturales y organizadas en forma de tramas han exhibido un fuerte dinamismo en países del Mercosur. En el marco de la evolución de los mercados mundiales, los nuevos modelos de apertura y la globalización, este dinamismo las convierte en ejes de acumulación y de crecimiento económico a partir de su inserción en el comercio internacional. Una parte importante de tal dinamismo productivo se asocia con la forma de organización adoptada — la trama—, que facilita el proceso de generación y la adopción de innovaciones.

En las principales tramas agroalimentarias de la región, un número reducido de grandes empresas, en su mayoría de capital transnacional, ha alcanzado en los últimos años posiciones jerárquicas de importancia. Estas empresas tienden a establecer grados crecientes de control a través del dominio de algunas tecnologías principales en el marco de una tendencia hacia la conformación de paquetes tecnológicos. Aun mediatizada por la presencia pública en algunas áreas, el dinamismo de estas grandes empresas reordena las jerarquías previas en las tramas, estableciendo nuevos equilibrios de poder internos.

Esta situación no es neutra desde varias perspectivas; no lo es, en particular, desde el ángulo de la acumulación regional, toda vez que dichas empresas conforman sus estrategias globales con objetivos que no siempre coinciden con la visión (estratégica) nacional o regional. cales, de aquellos nodos críticos con capacidad de redirigir hacia el mercado local los procesos de acumulación.
Este nuevo escenario - apertura económica en los mercados de bienes, capital y tecnología, configuración regional de las tramas, reordenación de las jerarquías internas a favor de empresas transnacionales y mayor peso del capital privado en la generación y difusión de innovaciones- lleva a reformular las políticas públicas. Siendo uno de sus objetivos el fortalecimiento de las capacidades locales/regionales de acumulación, es preciso redefinir tanto el propósito como la instrumentación de las políticas públicas, en función de un sentido estratégico de mayor alcance. Por lo tanto, al formular las políticas públicas habrá que considerar la estructura y dinámica de funcionamiento de las tramas (sus jerarquías, sus tecnologías principales, los nodos de sus sistemas de difusión), y será necesario que se diseñen instrumentos específicos con un mayor efecto de derrame a lo largo de ellas. También deberá replantearse necesariamente parte de la institucionalidad pública anterior, especialmente en las áreas tecnológicas. Las futuras políticas dedicadas a establecer o fortalecer la actividad de las tramas deberán hacer hincapié en la formulación de criterios estratégicos encaminados a lograr que una parte importante de los excedentes sea canalizada hacia los espacios locales o regionales donde estas organizaciones tengan asiento, en el marco de una tendencia creciente a generar mayor valor agregado local y a establecer formas consensuadas, sustentables y equitativas para su reparto.

Bibliografía

Albu, M. (1999): Technological Learning and Innovation in Industrial Clusters in the South, Electronic Working Paper Series, $\mathrm{N}^{\circ} 7$, Sussex, SPRU, Universidad de Sussex.

Aspiazu, D. y E. Basualdo (2000): El complejo vitivinícola argentino en los noventa. Obstáculos y potencialidades, Santiago de Chile, CEPAL.

Bisang, R. (2003a): Apertura económica, innovación y estructura productiva: la aplicación de la biotecnología en la producción agrícola pampeana argentina, Desarrollo económico, $\mathrm{N}^{\circ} 171$,
Buenos Aires, Instituto de Desarrollo Económico y Social (IDES), octubre-diciembre.

(2003b): Diffusion Process in Networks: the Case of Transgenic Soybean in Argentina, documento presentado en la primera Conferencia de Globelics, Río de Janeiro, noviembre.

(2003c): La trama de la carne bovina en Argentina, Buenos Aires, Comisión Económica para América Latina y el Caribe (CEPAL)/Banco Interamericano de Desarrollo (BID)/ Ministerio de Economía. 
(2004): Innovación y estructura productiva: la biotecnología en el agro pampeano, en A. Bárcena, J. Katz y otros (comps), Los transgénicos en América Latina: un debate abierto, LC/G.2227-P, Santiago de Chile, CEPAL. Publicación de las Naciones Unidas, $\mathrm{N}^{\circ}$ de venta: S.04.II.G.74.

Bisang, R. y G. Gutman (2001): Agrofood Networks and Regional Accumulation Processes: Which is the Institutional Framework Needed?, documento presentado en la III International Conference on Agrifood Chain-Networks Economics and Management, São Paulo, Universidad de São Paulo, noviembre.

Bisang, R., G. Gutman y otros (2000): La oferta tecnológica de las principales cadenas agroindustriales en el MERCOSUR amplia$d o$, Proyecto global "Organización y gestión de la integración tecnológica agropecuaria y agroindustrial en el Cono Sur", Montevideo, Programa Cooperativo para el Desarrollo Tecnológico Agropecuario del Cono Sur (PROCISUR)/Banco Interamericano de Desarrollo (BID).

Bortoleto, E. y J. Wilkinson (2000): Trajetoria e demandas tecnológicas nas cadeias agroalimentares do Mercosul ampliado. Lácteos; Proyecto global "Organización y gestión de la integración tecnológica agropecuaria y agroindustrial en el Cono Sur", Montevideo, Programa Cooperativo para el Desarrollo Tecnológico Agropecuario del Cono Sur (PROCISUR)/Banco Interamericano de Desarrollo (BID).

Buxedas, M. (2003): La estructura del comercio internacional de carne vacuna. Circuitos del comercio y dinámica del sector, Montevideo, CECUM.

Cap, E. (1997): Tecnologías agropecuarias con características de bienes semipúblicos, documento de trabajo, $\mathrm{N}^{\circ} 2$, Washington, D.C., Fondo Regional de Tecnología Agropecuaria, Banco Interamericano de Desarrollo (BID).

CEPAL (Comisión Económica para América Latina y el Caribe) (1995): Las relaciones agroindustriales y la transformación de la agricultura, LC/L.919, Santiago de Chile.

(2002): Componentes macroeconómicos, sectoriales y microeconómicos para una estrategia nacional de desarrollo. Lineamientos para fortalecer las fuentes de crecimiento económico, Estudios agroalimentarios, Buenos Aires, <www.cepal.org.ar>

CNPq (Consejo Nacional de Desarrollo Científico y Tecnológico) (1998): Agronegocio brasilero, Brasilia.

Dirven, M. (1999): Dairy clusters in Latin America in the context of globalization, International Food and Agribusiness Management Review, vol 2, $\mathrm{N}^{\circ} 3 / 4$, Oxford, Pergamon.

Granovetter, G. (1985): Economic action and social structure: the problem of embeddedness, American Journal of Sociology, vol. 91, $\mathrm{N}^{\circ} 3$, Chicago, The University of Chicago Press, junio.

Gutman, G. (1999a): El sector agropecuario y el sistema alimentario. Nuevas dinámicas, nuevos enfoques, Revista argentina de economía agraria, vol. 2, $\mathrm{N}^{\circ}$ 2, Buenos Aires, Asociación Argentina de Economía Agraria.

(1999b): Desregulación, apertura comercial y reestructuración industrial. La industria láctea en Argentina en la década de los noventa, en D. Aspiazu, G. Gutman y A. Vispo, $L a$ desregulación de los mercados. Paradigmas e inequidades de las políticas del neoliberalismo: las industrias lácteas, farmacéuticas y automotriz, Buenos Aires, Grupo Editorial Norma/Facultad Latinoamericana de Ciencias Sociales (FLACSO).

(2000): Trayectoria y demandas tecnológicas de las cadenas agroindustriales en el MERCOSUR ampliado. Oleaginosas: soja y girasol, Proyecto global "Organización y gestión de la integración tecnológica agropecuaria y agroindustrial en el Cono Sur", Montevideo, Programa Cooperativo para el De- sarrollo Tecnológico Agropecuario del Cono Sur (PROCISUR)/ Banco Interamericano de Desarrollo (BID).

(2002): Impact of the rapid rise of supermarkets on dairy product systems in Argentina, Development Policy Review, vol. 20, $\mathrm{N}^{\circ}$ 4, Oxford, Reino Unido, Blackwell Publishing.

Gutman, G. y P. Lavarello (2003): La trama de oleaginosas en Argentina, Documento B-3, Estudio 1.EG.33.7, Buenos Aires, Oficina de la CEPAL en Buenos Aires.

Gutman, G., E. Guiguet y P. Lavarello (2004): Ciclos de la lechería argentina. Una visión integradora de la dinámica macroeconómica y sectorial, Revista argentina de economía agraria, vol. 7, № 2, Buenos Aires, Asociación Argentina de Economía Agraria.

Gutman, G. y V. Cesa (2002): Innovación y cambio tecnológico en las industrias de la alimentación en Argentina, en R. Bisang, G. Lugones y G. Yoguel (comps.), Apertura e innovación en Argentina, Buenos Aires, Editorial Miño y Dávila, octubre.

Humphrey, J. y H. Schmitz (2001): Governance in global value chains, en G. Gereffi y R. Kaplinsky (comps.), IDS Bulletin, vol. 32, N 3 , Sussex, Reino Unido, Instituto de Estudios para el Desarrollo, Universidad de Sussex.

INTA (Instituto Nacional de Tecnología Agropecuaria) (2003): La cadena de la vid y los vinos, Mendoza, Centro Regional Cuyo, marzo.

Lavarello, P. (2003): La trama de maíz en Argentina, Buenos Aires, Comisión Económica para América Latina y el Caribe (CEPAL)/Banco Interamericano de Desarrollo (BID)/Ministerio de Economía.

Lindarte, E. (1994): Los institutos de investigación agrícola, Montevideo, Programa Cooperativo para el Desarrollo Tecnológico Agropecuario del Cono Sur (PROCISUR).

Paulino, C. y otros (2004): Competitividad de las cadenas agroindustriales en el MERCOSUR ampliado: carne, oleaginosas y aceites, textiles y algodón, Montevideo, Centro de Investigaciones Económicas (CINVE).

PROCISUR (Programa Cooperativo para el Desarrollo Tecnológico Agropecuario del Cono Sur) (2002): Los institutos nacionales de investigación agropecuaria del Cono Sur: nuevos ambientes y cambios institucionales, Montevideo, abril.

PROCISUR/BID (Programa Cooperativo para el Desarrollo Tecnológico Agropecuario del Cono Sur/Banco Interamericano de Desarrollo) (2000): Trabajos finales del Proyecto global. "Organización y gestión de la integración tecnológica agropecuaria y agroindustrial en el Cono Sur", en <www. procisur.org.uy>

Ramos, J. (1998): Una estrategia de desarrollo a partir de complejos productivos (clusters) en torno a los recursos naturales, LC/R.1743/Rev.1, Santiago de Chile, Comisión Económica para América Latina y el Caribe (CEPAL).

SEPCyT (Sistema de Evaluación de Proyectos Científicos y Tecnológicos) (2003): Segunda Encuesta Nacional de Innovación y Conducta Tecnológica de las Empresas Argentinas, Estudios, $\mathrm{N}^{\circ}$ 33, Buenos Aires, Sistema de Evaluación de Proyectos Científicos y Tecnológicos (SЕPCyT)/Instituto Nacional de Estadística y Censos (INDEC).

Wilkinson, J. (2002): The final food industry and the changing face of the global agrofood system, Sociologia ruralis, vol. 2, $\mathrm{N}^{\circ}$ 4, Oxford, Reino Unido, Blackwell Publishing.

Zylbersztajn, D. y E. Farina (1997): Agri-system management: recent developments and applicability of the concept, First Brazilian Workshop on Agri Chain Management, São Paulo, Programa de Estudos dos Negócios do Sistema Agroindustrial (PENSA).

Zylbersztajn, D. y C. Pinheiro Machado (2000): Competitiveness of meat agribusiness chain in Brazil and extensions for Latin America, São Paulo. 